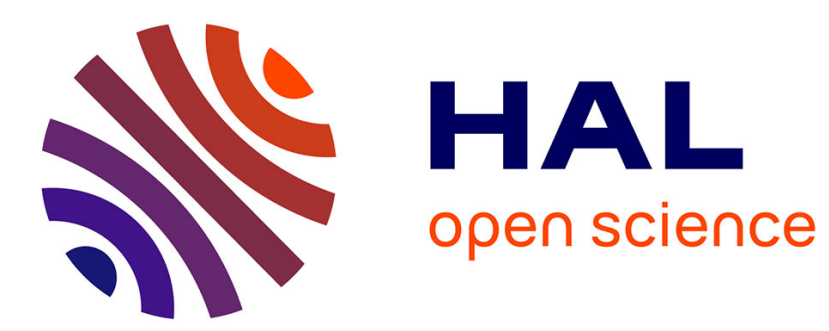

\title{
LA GESTION DES EXPLOITATIONS AGRICOLES : UN ÉTAT DES LIEUX DE LA RECHERCHE EN FRANCE
}

Philippe Jeanneaux, Hélène Blasquiet-Revol

\section{- To cite this version:}

Philippe Jeanneaux, Hélène Blasquiet-Revol. LA GESTION DES EXPLOITATIONS AGRICOLES : UN ÉTAT DES LIEUX DE LA RECHERCHE EN FRANCE. Gérer et Comprendre. Annales des Mines, 2012, Gérer et Comprendre, 107, pp.29-40. hal-02122219

\author{
HAL Id: hal-02122219 \\ https://hal.science/hal-02122219
}

Submitted on 7 May 2019

HAL is a multi-disciplinary open access archive for the deposit and dissemination of scientific research documents, whether they are published or not. The documents may come from teaching and research institutions in France or abroad, or from public or private research centers.
L'archive ouverte pluridisciplinaire HAL, est destinée au dépôt et à la diffusion de documents scientifiques de niveau recherche, publiés ou non, émanant des établissements d'enseignement et de recherche français ou étrangers, des laboratoires publics ou privés. 


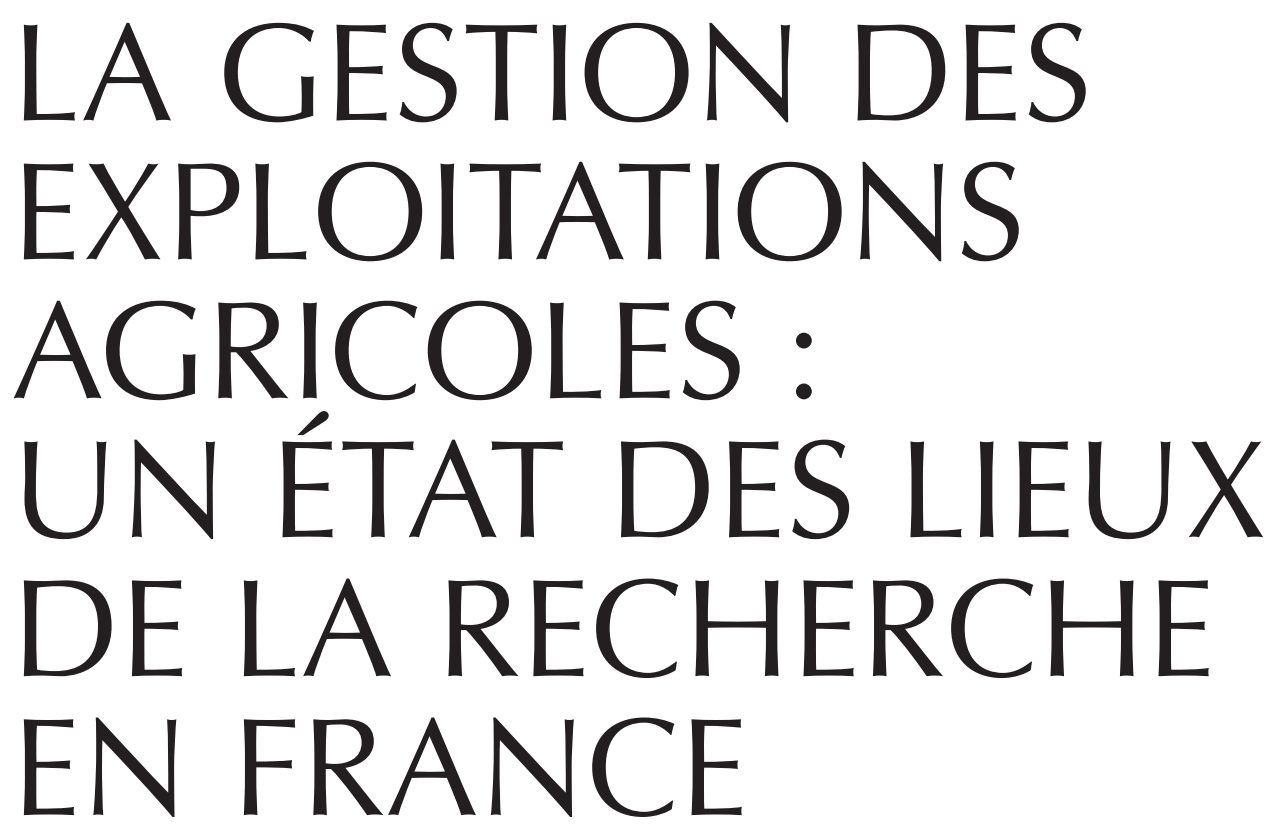

Le contexte économique des exploitations agricoles a fortement évolué ces dernières années, obligeant les agriculteurs à développer de nouvelles compétences et de nouveaux modes de gestion. L'ampleur de ces évolutions aurait pu se traduire par un renouvellement des travaux de recherche en gestion des exploitations agricoles. À travers l'étude des références bibliographiques de trois bases de données françaises recensant les travaux de recherche de l'Inra, nous avons réalisé un état des lieux de la production française de publications sur ce sujet depuis près de vingt ans (1990 à 2008). Nous avons observé une forte diminution du nombre des travaux scientifiques concernant la gestion des exploitations agricoles. Contrairement à nos suppositions et à l'état des travaux de recherche dans les pays anglo-saxons, le renouvellement des courants théoriques en gestion des exploitations agricoles n'a pas eu lieu. Les travaux sur le management stratégique restent marginaux, alors que cette démarche pourrait s'affirmer, à l'avenir, comme un levier essentiel de l'amélioration de la performance globale des exploitations agricoles.

Par Philippe JEANNEAUX* et Hélène BLASQUIET-REVOL**

* Professeur d'Économie Rurale.

** Ingénieur d'études. 


\section{INTRODUCTION : LES MUTATIONS DE L'AGRICULTURE ET LE BESOIN DE NOUVEAUX OUTILS DE PILOTAGE STRATÉGIQUE DES EXPLOITATIONS AGRICOLES}

Le contexte économique des exploitations agricoles a fortement évolué ces dernières années. La baisse tendancielle des prix, associée au maintien des charges, a provoqué une baisse tendancielle du revenu des exploitations agricoles. En réponse, les agriculteurs ont dû développer différentes stratégies afin de maintenir leur résultat agricole net par actif, en termes réels (voir l'Insee (1) - Compte de l'agriculture). D'un tion de leurs activités et de leurs revenus. Ces choix ont aussi eu tendance à professionnaliser les exploitations et à les inciter à opter de plus en plus pour un statut de société (GAEC, EARL, SCEA) (2).

\section{Les nouveaux contours de l'exploitation agricole}

Les exploitations agricoles ont tendance à s'éloigner du modèle de l'exploitation agricole familiale dite à deux UTH (3) pour se rapprocher d'un modèle d'entreprise (BARTHÉLÉMY, 1997 ; LAURENT, RÉMY, 2000 ; AMELINCKX, BARRAS, 2002).

Les contours traditionnels de l'exploitation agricole familiale éclatent, avec l'émergence de projets tech-

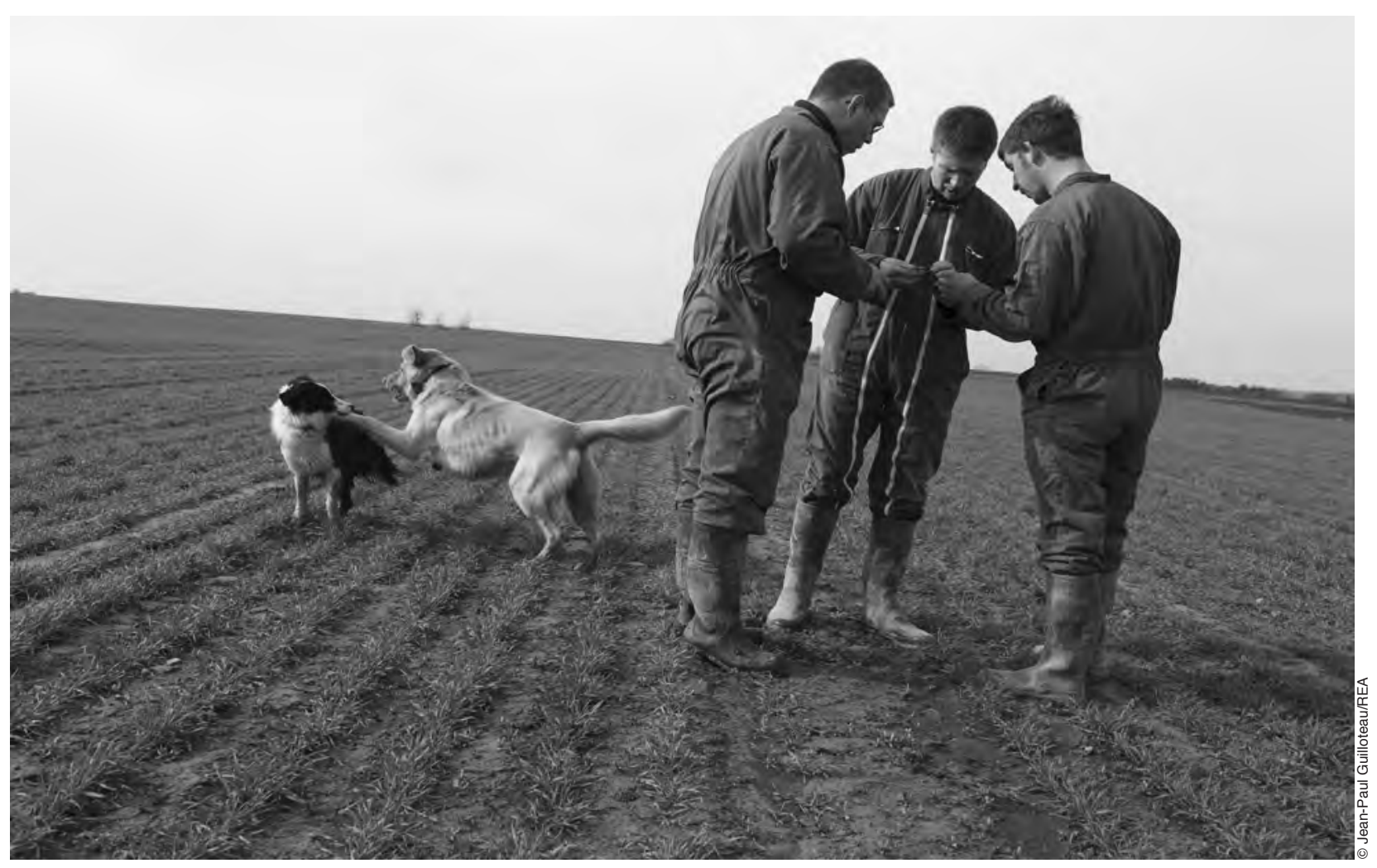

"Les exploitations agricoles ont tendance à s'éloigner du modèle de l'exploitation agricole familiale dite à deux UTH ("unités de travail humain") pour se rapprocher d'un modèle d'entreprise ". Agriculteurs sur un champ de céréales bio quiils exploitent en association dans le cadre d'un GAEC (groupement agricole d'exploitation en commun), Vendée, février 2011.

côté, la stratégie dominante a consisté à obtenir un avantage concurrentiel par la baisse des coûts de production, en cherchant à réaliser des gains de productivité via la spécialisation productive, l'agrandissement et la modernisation des structures de production. Et, de l'autre, les agriculteurs ont développé des stratégies de recherche d'un avantage concurrentiel par la différenciation (hors coût) ou par la diversifica-

(1) Résultat agricole net par actif en termes réels.

http://www.insee.fr/fr/themes/document.asp?reg_id=0\&ref_id=ip1247\#i nter4 niques, patrimoniaux et entrepreneuriaux (DROUET, BOULLET, 2006), dont la conduite exige parfois la création et la gestion de plusieurs sociétés emboîtées : groupement d'intérêt économique (GIE) pour commercialiser les produits ; société à responsabilité limitée (SARL) pour développer des activités de services extra-agricoles; groupement d'employeurs pour se partager les services d'un salarié ; société civile laitière

(2) GAEC : Groupement agricole d'exploitation en commun ; EARL : Exploitation agricole à responsabilité limitée ; SCEA : Société civile d'exploitation agricole.

(3) Unité de Travail Humain. 
(SCL) pour amortir plus rapidement l'achat d'un robot de traite; groupement foncier agricole (GFA) pour sécuriser et transmettre le parcellaire. L'exploitation agricole devient multipolaire et flexible (SÉrONIE, Boullet, 2007) et son périmètre s'avère de plus en plus difficile à cerner.

En parallèle à ces évolutions productives, structurelles et statutaires se développent de nouvelles formes de relations entre les exploitations et leurs environnements amont et aval. D'une logique de versement d'aides publiques sans exigence explicite d'une contrepartie de la part des agriculteurs, les politiques agricoles passent de plus en plus à une logique d'attribution d'aides conditionnée par le respect de la réglementation ou d'octroi d'une rémunération contractualisée. Ces dispositifs contractuels fonctionnent selon un principe qui oblige les agriculteurs à assurer de nouvelles tâches de conservation de l'information et de constitution d'éléments de preuve vis-à-vis de l'extérieur, ou à s'inscrire dans le cadre de la certification de l'exploitation (MAZÉ, AUBrY, PAPY, 2000 ; Grolleau, 2001).

Dans ce contexte, les agriculteurs doivent donc développer de nouvelles compétences pour adapter leurs exploitations à des évolutions structurelles et productives rapides. Leur capacité à penser le changement de leur exploitation, à estimer les risques que représentent des marchés agricoles de plus en plus fluctuants, à utiliser de manière stratégique l'information et, plus globalement, à agir dans un environnement davantage marqué par les incertitudes est sans doute de nature à modifier les pratiques de gestion de l'exploitation (ou, dit autrement, de son pilotage stratégique).

$\overline{\text { Changement et innovation dans les pratiques }}$ de gestion

Dans son ouvrage sur la gestion des exploitations agricoles publié en 1957, Paul-Henry Chombart de Lauwe proposait de transposer des méthodes et des outils de gestion de l'industrie aux exploitations agricoles. Il a ainsi proposé des indicateurs de performance permettant de caractériser les exploitations et de les inciter à améliorer leur profit. Mais aujourd'hui, les exploitations agricoles sont devenues flexibles; elles combinent plusieurs objectifs : entrepreneurial, patrimonial, technique et social. L'agriculteur, en plus de gérer sa production, de mầtriser ses coûts et de traiter le risque, doit gérer les questions environnementales, sociales et territoriales liées à son exploitation. Pour gérer cet ensemble d'éléments de l'environnement, qui vont modifier ses pratiques, et dans le but d'atteindre ses multiples objectifs, l'agriculteur doit à la fois anticiper et s'adapter. Le contexte actuel force les agriculteurs à repenser la gestion de leurs exploitations et à innover en imaginant de nouveaux indicateurs de performance.
La question de recherche

C'est à partir de ce postulat que nous avons supposé que la façon dont les agriculteurs traitent les enjeux actuels de l'agriculture (changement rapide de l'environnement, incertitude...) nécessite que soient définis de nouveaux indicateurs de performance et de nouveaux outils de pilotage stratégique de l'exploitation. Nous supposons que la production d'outils d'aide à la décision ou de méthodes de management stratégique devrait constituer un front de la recherche en économie agricole et en sciences de gestion, avant que ces outils soient transférés aux professionnels. Les démarches de management stratégique sont aujourd'hui fortement développées dans le secteur industriel (tant dans les grandes entreprises que dans les PME) et ont fait la preuve de leur pertinence. Par conséquent, nous posons la question de savoir comment les évolutions du contexte agricole et leurs effets sur le pilotage de l'exploitation agricole ont été appréhendés en France par les chercheurs spécialisés dans le domaine du fonctionnement des exploitations agricoles.

Dans la première partie de cet article, nous présenterons la méthodologie qui a été employée pour évaluer la production scientifique française en matière de gestion de l'exploitation agricole. Dans une deuxième partie, nous présenterons les résultats tirés de l'analyse des bases de données que nous avons étudiées sous la forme d'un état des lieux de cette production. Enfin, nous discuterons ces résultats, d'une part, au regard des fronts de recherches en train d'émerger et, d'autre part, au regard des différents courants théoriques de la gestion des exploitations agricoles.

\section{LE CADRE MÉTHODOLOGIQUE : UNE RECHERCHE DOCUMENTAIRE SUR LA PRODUCTION SCIENTIFIQUE CONSACRÉE À LA GESTION DES EXPLOITATIONS AGRICOLES}

\author{
$\overline{\text { Délimitation de l'objet de l'étude au moyen }}$ \\ de mots-clés
}

Nous avons identifié le champ disciplinaire principal qui selon nous aborde la gestion des exploitations agricoles et dont nous supposons qu'il est porteur de fronts de recherche.

Ce champ recouvre les disciplines de l'économie de la production agricole et celles des sciences de gestion. Ce champ s'inscrit dans la continuité des travaux de Chombart de Lauwe, puis de ceux de Marshall et Brossier (1981). D'autres travaux ont été menés par les spécialistes du management ou de la stratégie de la firme, qui ont largement inspiré les travaux des chercheurs anglo-saxons sur le farm management. 
Nous avons ensuite sélectionné plusieurs mots-clés généralement mobilisés par les chercheurs pour identifier et délimiter leurs productions scientifiques dans les bases de recensement des travaux de recherche. Les mots et expressions clés que nous avons retenus sont : gestion de production, stratégie, entreprise, management, analyse financière, prise de décision, aide à la décision, conseil et, enfin, gestion de l'exploitation agricole. Ces mots-clés permettent de recenser la littérature consacrée à la thématique de la gestion de l'exploitation agricole.

\section{Les espaces de diffusion des connaissances \\ sur la gestion de l'exploitation agricole}

Compte tenu de la délimitation de notre sujet, nous avons choisi de mener une recherche documentaire tant dans le domaine des sciences humaines et sociales que dans celui de l'agronomie à l'échelle de la France. Pour viser la représentativité de la recherche sur la gestion de l'exploitation en France depuis vingt ans, il nous a semblé pertinent de nous intéresser en priorité aux travaux de l'Inra (Institut National de la Recherche Agronomique). Nous avons donc choisi d'examiner les travaux recensés dans les bases de référencement des publications des chercheurs de l'Inra. Ce choix s'explique aussi par le fait que cette information est accessible à partir de trois bases de données (ProdINRA, BDESR et BDSAD) regroupant les travaux des chercheurs (voir l'encadré "Présentation des bases de données »).

\section{PRÉSENTATION DES BASES DE DONNÉES}

Les trois bases de données étudiées (ProdINRA, BDESR et BDSAD) sont intéressantes par deux aspects.

Premièrement, elles couvrent de nombreux champs thématiques correspondant de près ou de loin aux différentes approches de la gestion de l'exploitation agricole: production, marchés et échanges agricoles; transformation et distribution des produits agro-alimentaires; consommations alimentaires et risques ; environnement ; espaces ruraux et emploi ; et des recherches disciplinaires (sciences humaines et sociales - sciences économiques).

Deuxièmement, elles regroupent un grand nombre de références : 32795 depuis 1985 pour la BDESR, 4241 pour la BDSAD depuis 1987, et plus de 100000 depuis 2006 pour ProdINRA.

Du recueil des références recensées dans les bases de données à leur traitement

Nous avons réalisé une recherche documentaire portant sur des références françaises illustratrices du cas français, à partir de requêtes effectuées au moyen d'un ensemble de mots-clés (voir l'encadré "Recueil et mise en mémoire des données »).

\section{RECUEIL ET MISE EN MÉMOIRE DES DONNÉES}

La période retenue pour l'étude va de 1990 à 2008. La recherche sur les bases de données se fait par mots-clés représentatifs du champ de la gestion de l'exploitation agricole. Afin de couvrir entièrement et d'être exhaustif dans nos recherches sur ces bases de données, nous avons sélectionné sept mots-clés principaux recouvrant les différents champs que nous avons préalablement identifiés. L'interrogation s'est faite par combinaison des sept mots-clés identifiés en utilisant les opérateurs booléens classiques (et, ou, sauf...). Les requêtes ont permis de recenser de nombreuses références avec parfois des doublons qui ont été éliminés. La base, une fois "nettoyée ", contient 427 références. Nous avons réalisé des recoupements de requêtes afin de restreindre le nombre des références qui auraient pu nous échapper.

La recherche sur ces bases concerne différents documents : les articles de périodique, de magazines spécialisés, les ouvrages, les chapitres d'ouvrage, les mémoires, les thèses, les actes de colloque, les communications à des colloques, les rapports, et les chapitres de rapport. Sont exclus de cette recherche : brochures, plaquettes, brevets, posters, cartes, documents audiovisuels et notices techniques.

Aucun filtre de langue n'étant disponible sur les bases de données, la recherche par mots-clés a fait quelque fois ressortir des documents non publiés par des chercheurs français mais par des chercheurs espagnols, anglais, des documents publiés avec un chercheur de l'Inra et illustratifs de leur pays respectif. Ces documents sont ensuite éliminés lors du traitement des données puisqu'ils ne sont pas représentatifs du cas français.

La mise en mémoire des références recueillies a été réalisée via la construction d'une base de données spécifiques sous Excel et qui, pour chaque référence, renseigne : l'auteur, l'année de publication, le type de support, le titre du document et les mots-clés principaux associés au document dans les bases de données.

Des traitements en statistique descriptive et en analyse de données (analyse factorielle des correspondances multiples - AFCM ; classification ascendante hiérarchique - CAH) ont été réalisés avec le logiciel SPAD v.7.0 sur les références recueillies. Pour cela, nous avons procédé à un recodage des différentes données 


\begin{tabular}{|l|l|}
\hline \multicolumn{1}{|c|}{ Variable } & \multicolumn{1}{c|}{ Modalités } \\
\hline Période de publication & 4 classes : 1990-1992;1993-1995; 1996-1999; 2000-2008 \\
\hline Base de données & BDESR ; ProdINRA ; BDSAD \\
\hline Support de publication & $\begin{array}{l}\text { Revues ; Ouvrages ; Chapitres d'ouvrages ; Rapports ; Travaux } \\
\text { intermédiaires (communications, actes de colloques...) ; Thèses }\end{array}$ \\
\hline Échelle d'analyse & Micro ; Méso ; Macro ; Autre échelle \\
\hline Objet d'étude & Parcelle ; Entreprise ; Organisation ; Territoire ; Pays \\
\hline Type de travaux & Analyse ; Diagnostic ; Évaluation ; Simulation ; Outils ; Autre \\
\hline Sujet traité & $\begin{array}{l}\text { Aide à la décision ; Analyse financière ; Gestion de la production ; Management ; } \\
\text { Économie de la production }\end{array}$ \\
\hline Secteur & Agriculture ; Environnement ; Industries agro-alimentaires ; Rural \\
\hline
\end{tabular}

Tableau $n^{\circ} 1$ : Les variables d'analyse retenues et leurs déclinaisons en différentes modalités.

textuelles de notre base. Nous avons ainsi sélectionné huit variables d'analyse (voir le tableau $n^{\circ} 1$ ).

\section{LES RÉSULTATS : ÉTAT DES LIEUX DE LA PRODUCTION DE LA RECHERCHE FRANÇAISE EN GESTION DE L'EXPLOITATION AGRICOLE}

À partir de la recherche que nous avons effectuée sur les bases de données choisies et selon les mots-clés sélectionnés, 427 références (écrites par 564 auteurs différents) ont été répertoriées entre 1990 et 2008. Ces 427 références représentent moins de $1 \%$ de la production de l'Inra sur la période 1990-2008. Elles ne sont pas réparties de la même manière sur l'ensemble de cette période : après un pic du nombre des références en 1994, nous avons relevé une tendance à la diminution du nombre des références concernant la gestion de l'exploitation agricole entre 1996 et 2004, avant d'atteindre une certaine stabilité au cours de la dernière période (voir la figure 1 ci-dessous).

La diffusion des idées s'est faite à hauteur de $33 \%$ dans des revues, suivies des ouvrages et des rapports
(30\%) et de travaux intermédiaires $(26 \%)$ de types communications ou documents de travail et, enfin, des mémoires et thèses $(11 \%)$. Nous avons noté que la discipline dominante est l'économie (avec $39 \%$ des références), suivie par l'agronomie (29\%) et l'écologie $(11 \%)$. Les sciences de gestion ne représentent finalement que $21 \%$ des références.

Après ce regard général, nous avons affiné l'analyse en construisant une typologie de la production scientifique selon quatre classes.

La première classe, que nous avons intitulée "Économie de la production et gestion de l'exploitation agricole ", compte 120 références et rassemble une majorité de productions publiées en début de période (1990-1992). Il s'agit de travaux fondés sur une approche de l'économie de la production agricole ou sur l'analyse de la gestion de l'exploitation agricole.

On y trouve les recherches portant sur l'aide à la décision, l'analyse financière, le management ou encore la stratégie des exploitations agricoles. Cette classe est à rattacher aux enjeux économiques auxquels les exploitations agricoles ont fait face en développant une stratégie de recherche de l'avantage concurrentiel par la

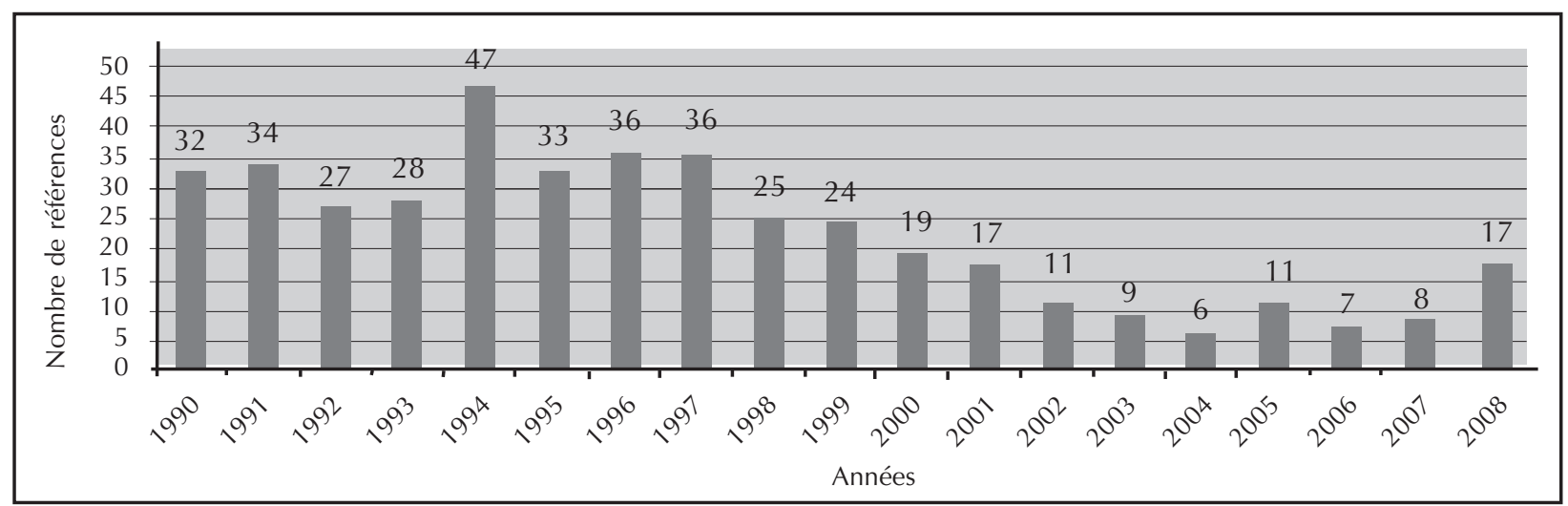

(Source : BDESR, BDSAD et ProdINRA)

Figure 1 : Évolution du nombre des références d'ouvrages traitant de la gestion de l'exploitation agricole, entre 1990 et 2008. 
baisse des coûts, qui a sans doute amené les chercheurs à analyser et à accompagner cette orientation dominante. Les travaux de cette classe s'axent autour de la modélisation, de la simulation et des outils d'aide à la décision. L'avènement de l'informatique (dans les années 1990) a orienté les recherches portant sur le renouvellement du pilotage des exploitations agricoles (logiciels, systèmes informatisés d'aide à la décision ...) et leur analyse.

Ces travaux s'inscrivent dans la filiation de l'approche standard, mais ils proposent aussi de revisiter la rationalité des agents et la façon dont les agri- classe contient, en premier lieu, des travaux traitant des effets de la politique agricole commune (PAC) sur les exploitations. Elle est, en second lieu, marquée par de nombreux travaux traitant des stratégies de diversification (agritourisme...) et de différenciation (AOC, labels...). Les productions de cette classe reposent majoritairement sur des travaux réalisés par des étudiants (mémoires et thèses).

La troisième classe s'intitule "Dynamique des espaces ruraux». Comptant 103 références, elle rassemble des travaux touchant au rural et étudiant plus particulièrement la dynamique des espaces ruraux. C'est

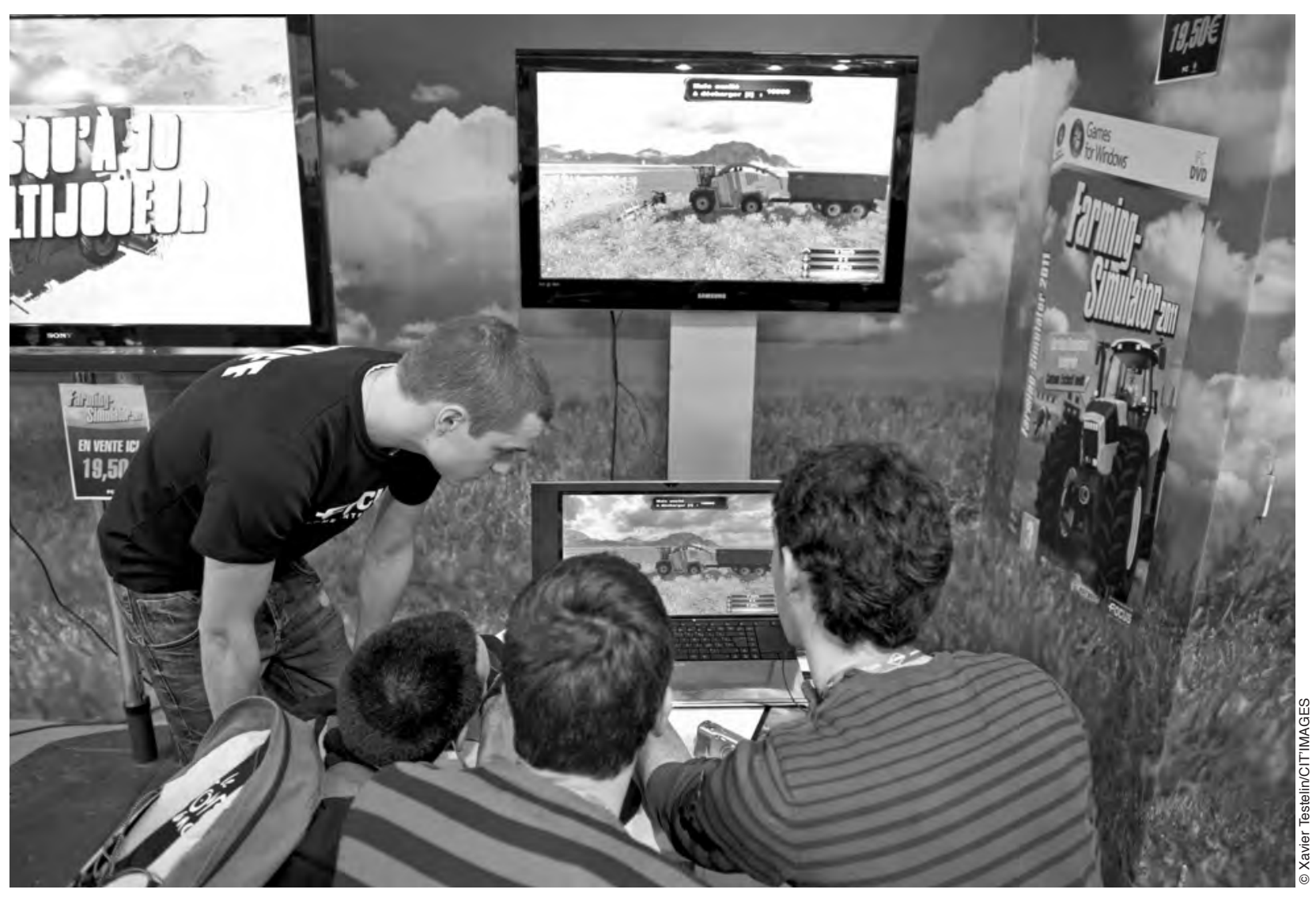

"L'avènement de l'informatique dans les années 1990 a orienté les recherches portant sur le renouvellement du pilotage des exploitations agricoles (logiciels, systèmes informatisés d'aide à la décision ...) et leur analyse ". Jeu vidéo permettant de simuler la gestion d'une exploitation agricole, Salon de l'Agriculture, Paris, mars 2011.

culteurs prennent leurs décisions. Nous pouvons citer, comme exemples de cette classe, les travaux de Brossier et al. (1990), Chatelin et POUSSIN (1991), Colson et Pineau (1991) ou bien encore HÉmidy et Soler (1992), qui s'inscrivent dans ce front de recherche.

Nous avons intitulé notre deuxième classe de travaux "Socio-économie agricole " (cette classe comporte 96 références). Elle regroupe des travaux (diffusés pour la majorité d'entre eux entre 1993 et 1995) dont l'objet d'étude est d'échelle intermédiaire (organisation, territoire...). Le secteur de ces références est à rattacher au rural et aux industries agro-alimentaires. Cette dans cette classe que se retrouvent les productions sur la multifonctionnalité de l'agriculture, des travaux qui se densifient, autour de l'année 1999, avec la promulgation de la loi d'orientation agricole $\mathrm{n}^{\circ} 99-574$ du 9 juillet 1999 . On y trouve de multiples travaux portant sur l'agriculture biologique (AB) (BENOÎT, 2001), l'environnement (HUBERT, 1999 ; Baudry et Thenail, 2001) et certaines mesures agro-environnementales (THANN-BERGER, 1999 ; RÉMY, 2002). Dans les années 2000, la thématique de la multifonctionnalité a ensuite été prolongée par les travaux portant sur la question de la durabilité des exploitations agricoles. 
Enfin, la quatrième classe, que nous avons intitulée "Agronomie et gestion de la production agricole", comprend 108 références et rassemble les travaux étudiant avant tout la conduite des parcelles ou celle des troupeaux. Ces travaux sont particulièrement abondants durant la période 2000-2008. Ils correspondent au champ de l'agronomie des systèmes techniques et, bien que relevant selon nous d'une branche des sciences de gestion, ils ne sont pas recensés comme tels par les agronomes. Ils sont essentiellement agricoles, à une échelle micro (parcelle, troupeau...). Cette classe concerne des travaux de type évaluatif publiés sous la forme de travaux intermédiaires (communications, rapports...) et abordant la gestion technique de l'exploitation du système de production.

\section{DISCUSSION : UN RENOUVELLEMENT THÉORIQUE INCOMPLET DE LA GESTION DE L'EXPLOITATION AGRICOLE EN FRANCE}

Tout d'abord, force est de constater que la délimitation des articles par mots-clés n'est pas très satisfaisante, car nous avons finalement recueilli de très nombreuses références qui étaient hors champ. En effet, de nombreuses références correspondent à des travaux recensés dans les catégories d'études en "Socio-économie agricole" et en "Dynamique des espaces ruraux " menés à des échelles plus larges que l'échelle, micro, de l'exploitation agricole.

Enfin, notre démarche éclaire la question de l'état des connaissances autour de la gestion de l'exploitation agricole, qui a été peu étudiée jusqu’à présent. Nous avons toutefois relevé quelques rares ouvrages qui établissent l'état des courants théoriques autour de la gestion de l'exploitation agricole, comme l'ouvrage de Brossier et al. (1997), ou autour de la gestion de la production dans le champ des sciences agronomiques (COLENO et DURU, 2005).

\section{L'évolution des différents courants de la gestion des exploitations agricoles}

Revenons sur les courants théoriques qui ont été le creuset des pratiques de gestion de l'exploitation et de l'économie de l'exploitation en France depuis cinquante ans et mettons-les en regard des résultats obtenus dans le cadre de notre recherche. Pour ce faire, nous nous proposons de nous centrer sur les travaux de gestion de l'exploitation agricole repérés au sein des catégories "Agronomie et gestion de la production agricole " et "Economie de la production et gestion de l'exploitation agricole». En effet, les deux autres catégories de travaux (les classes 2 et 3 ) n'apportent pas d'éléments d'analyse pertinents pour notre recherche.
Les travaux de la catégorie «Économie de la production et gestion de l'exploitation agricole » (classe 1)

Les documents identifiés dans le cadre de cette recherche sont très fortement marqués par le courant de la théorie néoclassique et celui de la théorie de la prise de décision. Le courant de la gestion se situant, quant à lui, dans la filiation de la théorie néoclassique de la firme, est une approche normative qu'a développée Chombart de Lauwe (1963) et qui vise à fournir les bons outils de gestion devant permettre de réaliser la meilleure allocation des ressources et de maximiser le profit. Elle s'inscrit dans la filiation des théories néoclassiques de l'entreprise (TNCE, ou production economics, selon Colin, 1990). La gestion se définissant comme "l'art des combinaisons rentables" aux yeux de Chombard de Lauwe, celui-ci considère explicitement la gestion comme un moyen d'industrialiser l'agriculture pour la sortir de sa sous-productivité. Laccent est mis sur des outils classiques de gestion issus de la comptabilité qui permettent d'élaborer des références et des normes technico-économiques à suivre (analyse de groupe, marges brutes, coûts de production...). La gestion de l'exploitation s'est aussi enrichie de travaux sur l'analyse économique et financière, avec la méthode des flux économiques et financiers. Ces travaux ont été réalisés dans l'optique d'une amélioration des approches du diagnostic économique et financier de l'exploitation, là encore avec cette idée de transposer à l'agriculture les méthodes d'analyse de l'industrie, dans la filiation de Chombart de Lauwe. La firme, dans la théorie néoclassique, est considérée comme un tout, comme une boîte noire omnisciente. Comme le fait remarquer Colin (1990), cette théorie ne permet pas de rendre compte des problèmes de l'entreprise et du comportement de l'agriculteur (homo economicus), qui ne chercherait, selon cette théorie, qu’à maximiser son profit.

En réaction à cette vision néoclassique par trop réductrice du fonctionnement de l'exploitation agricole se sont développés des travaux autour de la prise de décision et de l'exploitation vue comme un système piloté. L'agriculteur ne cherche plus, dès lors, seulement à maximiser son profit; il cherche d'abord à atteindre une situation satisfaisante, qui ne correspond pas nécessairement à un optimum économique. La théorie systémique appliquée à l'entreprise place l'action au centre de son objet scientifique. L'autonomie d'action est vue comme la capacité des acteurs à s'organiser et à inventer des solutions aux problèmes qui surgissent. "L'entreprise ne s'organise pas pour produire, elle s'organise pour résoudre les problèmes qu'elle perçoit, et donc pour décider de ses comportements. " (MARSHALL et al., 1994). Cette grande innovation dans la gestion de l'exploitation agricole en France trouve son origine dans les travaux du Groupe des Dijonnais. 
Plusieurs chercheurs associés à l'Inra Sad (Systèmes Agraires \& Développement, à l'époque) et/ou à l'Inrap (Institut National de Recherches et d'Applications Pédagogiques) ont ainsi pu développer un nouveau regard sur l'exploitation agricole et son pilotage. Au cours des décennies 1980 et 1990, cette approche va modifier la démarche des conseillers agricoles, qui vont dès lors développer un nouvel accompagnement des agriculteurs. De nouveaux outils font également leur apparition, tel que le budget partiel (qui vise à prendre en compte la notion de coût d'opportunité). Ce courant a eu de nombreux impacts (davantage sur le plan pédagogique que dans le domaine de la recherche).

Enfin, au cours de la décennie 1990, quelques rares travaux originaux en gestion de l'exploitation agricole ont introduit la question de la stratégie de l'entreprise et du management stratégique, en cherchant à adapter celuici à l'exploitation agricole. Ces travaux conduits par Hémidy et Soler (1992, 1994), Guichard et Michaud (1994) ou encore Marchesnay $(1994,1997)$ ont mis en évidence la spécificité du modèle stratégique de la très petite entreprise, à laquelle se rattache l'exploitation agricole. Ce courant autour de l'analyse stratégique de l'exploitation est resté marginal. Quelques travaux ont cependant été développés autour de l'amélioration continue de la qualité, ou autour de l'environnement (Grolleau, 2001).

Les travaux de la catégorie « Agronomie et gestion de production agricole» (classe 4)

Il nous semble que le champ de l'agronomie et de la zootechnie des systèmes techniques doit être abordé dans cette analyse tant celui-ci semble être le plus actif en matière d'analyse $\mathrm{du}$ comportement des agriculteurs. Ces travaux, en grande majorité techniques et réalisés par des "agronomes des sciences sociales ", constituent une constante dans l'étude de la gestion de l'exploitation agricole. Ce champ de l'agronomie des systèmes techniques recouvre les travaux des agronomes portant aussi bien sur le pilotage global de l'exploitation ou, plus précisément, le pilotage du système de production (GIRARD et al., 1994 ; DURU, 2008) que sur le pilotage d'activités, telles que l'irrigation (DEBAEKE et al., 2008). Il semble que cette catégorie d'agronomes doive être rattachée à celle des spécialistes de la gestion de la production (qui est une branche des sciences de gestion) davantage qu'à celle des agronomes des sciences de la vie et de la terre.

Au final, tous ces travaux donnent l'impression d'un découpage fonctionnel de la gestion de l'exploitation. D'un côté, nous trouvons l'analyse économique et financière, avec une conception de la gestion rattachée à la maîtrise de la lecture des documents comptables et des indicateurs de performances qui en sont tirés. Ce courant est en déclin et n'a pas été relancé par celui du management stratégique. D'un autre côté, nous trouvons les analyses des agronomes, qui développent des travaux en gestion de production centrés sur la conduite des ateliers, sur le pilotage d'une technique ou encore sur l'organisation du travail.

\section{Le farm management: assisterait-on à I'apparition} d'un nouveau courant?

Au regard de nos premières investigations, force est de constater que la gestion de l'exploitation agricole est un domaine de recherche principalement traité par les économistes et les agronomes. Pourtant, actuellement, la gestion de l'exploitation agricole en France est étudiée moins par les économistes que par les agronomes. Les années 2000 représentent d'ailleurs une rupture dans le domaine de la gestion de l'exploitation agricole. Quelles que soient les disciplines, les publications chutent fortement. Cette diminution semble au premier abord refléter un désintérêt pour l'agriculture, de manière générale, et pour la gestion de l'exploitation agricole, en particulier. On comprend mieux cette évolution quand on consulte le schéma stratégique (1999-2003) du département "Économie et sociologie rurale " (ESR devenu SAE2) de l'Inra (INRA, 1999). Il affiche explicitement sa décision d'abandonner les recherches en gestion des exploitations agricoles, une décision motivée par des difficultés de divers ordres: une problématique de recherche difficilement valorisable sur le plan académique, un faible effectif de chercheurs en gestion et l'absence de cadres scientifiques pour encadrer des doctorants ou de jeunes recrues. Les crises alimentaires de la période 2007-2009 semblent toutefois avoir ravivé l'intérêt pour les questions agricoles, sans que l'on sache si ce nouveau contexte suscitera un renouvellement des approches de gestion. Les publications sur l'analyse financière des exploitations et sur la prise de décision (classe 1) ont diminué, voire totalement disparu (voir la figure 2). Seules les questions environnementales et les questions de qualité des produits ont trouvé un écho dans les quelques travaux consacrés au management stratégique de l'exploitation agricole.

Cette rupture intervenue dans les années 2000 ne se retrouve pas dans les pays anglo-saxons, où l'étude de la gestion des exploitations agricoles a su se renouveler à travers le farm management. En France, le management stratégique de l'exploitation agricole a été abordé timidement à partir de 1994 à travers les travaux de certains auteurs (comme Hémidy). Ces questions sont classiquement traitées par les spécialistes de la gestion d'entreprise en termes de stratégie et de management (au sens de pilotage) stratégique de la firme. Nous faisons l'hypothèse que si les travaux sur le management stratégique sont restés marginaux, c'est parce que les agriculteurs (grâce à la Politique Agricole Commune - PAC) étaient protégés contre des contextes de concurrence exacerbée entre firmes. 


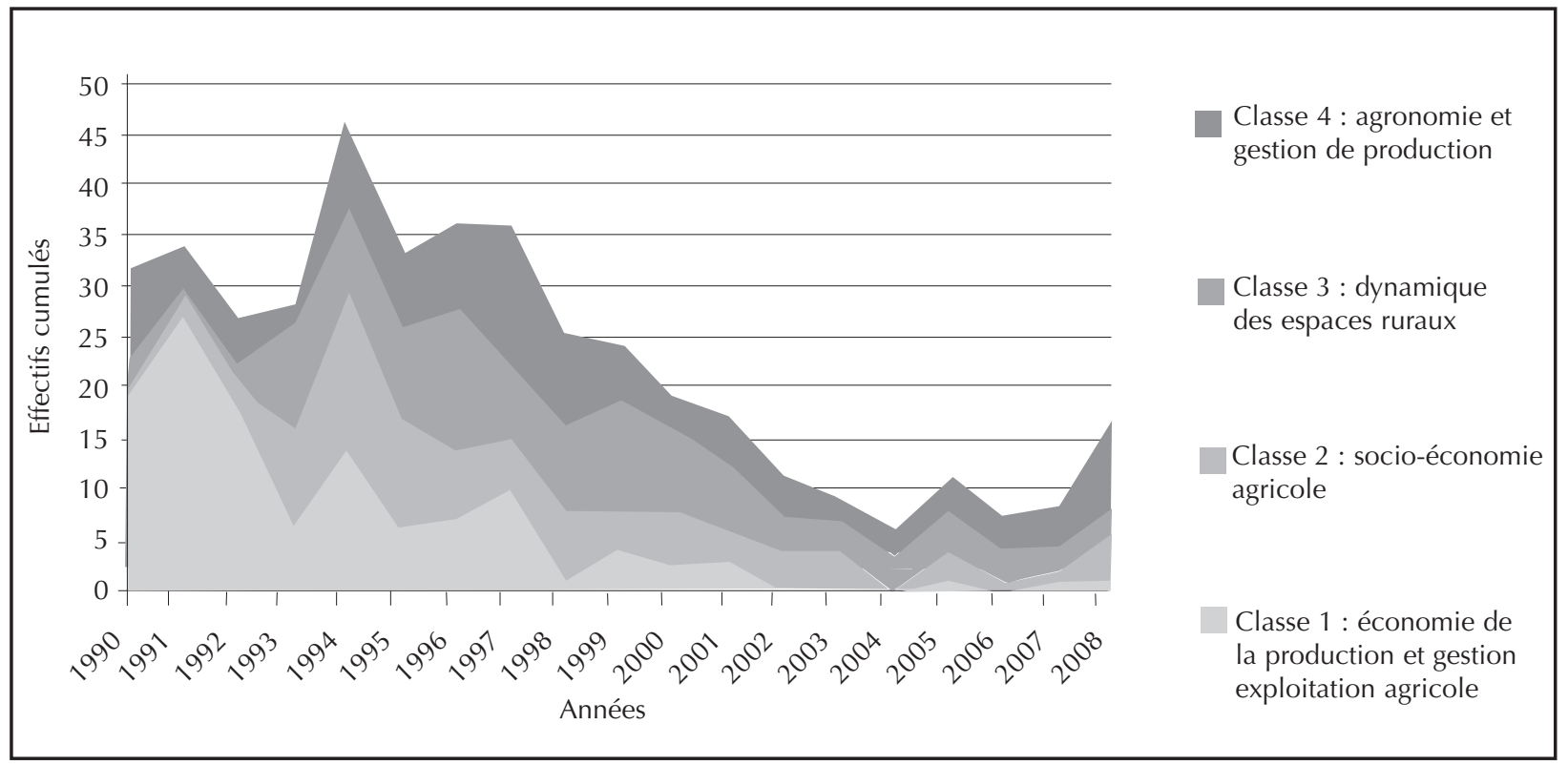

Figure 2 : Évolution des effectifs des quatre classes des travaux recensés.

Désormais, dans un contexte de dérégulation, les exploitations agricoles risquent de devoir de plus en plus s'inscrire dans des démarches de management stratégique en vue d'améliorer leurs performances économiques, sociales, techniques et environnementales - une situation que la recherche française en économie agricole ne semble pas avoir intégrée.

Le farm management tient compte de tous les aspects du management classique, mais s'applique tout particulièrement aux exploitations agricoles. Les exploitations ne sont plus gérées au quotidien, mais pilotées pour le futur, dans une perspective de durabilité et d'amélioration continue de leur performance globale. Nos investigations bibliographiques à l'échelle internationale montrent aussi qu'une littérature abondante existe en langue anglaise sur la démarche d'un management stratégique adapté à l'exploitation agricole (une quinzaine d'ouvrages, depuis le début des années 1980, traite du farm management: Barnard, Nix, 1979 ; Casavant, Infanger, 1999 ; Kay, 1986 ; Olson, 2004; Turner, Taylor, 1998, pour ne citer que quelques auteurs), une littérature qui n'a pas d'équivalent en langue française. Tous ces auteurs reprennent quasi-systématiquement le diagramme type des fonctions du management, auxquelles ils intègrent les concepts de l'économie de la production agricole et de la prise de décision.

La démarche de management stratégique repose sur plusieurs étapes successives:

1) la planification de la stratégie, qui passe par la définition et la formalisation d'une politique générale, déclinée ensuite en objectifs stratégiques et en orientations d'action visant la construction d'un plan d'action ;

2) la mise en ouvre dudit plan d'action dans l'optique de progresser en passant de la situation actuelle vers la situation projetée ;
3) le contrôle des résultats obtenus, qui permet ensuite d'évaluer l'atteinte des performances souhaitées et le plan d'action ;

4) La ré-interrogation de la stratégie au regard de l'évaluation, qui doit favoriser l'adaptation des objectifs stratégiques, si besoin est, ainsi que la modification du plan d'action.

Cette démarche est sans doute implicite, chez les agriculteurs, et elle est donc finalement peu formalisée. Dans ces travaux anglo-saxons, le management stratégique est présenté comme une démarche de progrès pouvant être assimilée au processus d'amélioration continue que l'on trouve dans les démarches de management certifiées ISO 9001 ou ISO 14001. Justifié au départ par la nécessité de mettre en évidence le fait que les écarts de performance résultent du management de la performance économique, le système de management tel que le proposent les travaux anglo-saxons a pris en compte, petit à petit, le fait que les agriculteurs ont des objectifs multiples et parfois contradictoires, en raison d'environnements très variables. Nous savons aussi que les approches de planification stratégique font l'objet de nombreuses critiques (MinTZBERG, 1994). On leur reproche d'être très analytiques, descendantes, focalisées sur la mesure quantitative de ratios très souvent financiers, et de faire perdre de vue la vision systémique de la stratégie en se focalisant sur une dimension opérationnelle qui se traduit par un contrôle excessif au travers d'indicateurs excessivement nombreux. Ce ne sont toutefois pas ces limites qui peuvent expliquer le désintérêt des chercheurs pour cette thématique du management stratégique, ces limites ayant par ailleurs été intégrées, d'une certaine manière, avec l'adoption des tableaux de bord stratégiques (Balanced ScoreCard: BSC) (KAPLAN et 
NORTON, 1992). Cette dernière approche correspond à une représentation de la stratégie et de sa mise en ouvre qui pourrait s'inscrire dans l'école de l'apprentissage (MinTZBERG, 1994). Cette approche du management stratégique recourant aux tableaux de bord stratégiques (et prospectifs) permet d'avoir une vision globale d'un projet d'entreprise piloté par quelques critères stratégiques représentant des objectifs à atteindre. La démarche BSC a par ailleurs été appliquée à l'exploitation agricole (NOELL et LUND, 2002) : cette expérience montre que des travaux de recherche portant sur l'élaboration de la stratégie de l'exploitation agricole et sur sa mise en œuvre occupent aussi un des fronts de la recherche en sciences de gestion.

Nous constatons que le renouvellement des courants théoriques n'a été que partiel, en France, en matière de gestion de l'exploitation agricole. Cette incomplétude peut renvoyer au fait que l'attention des chercheurs s'est énormément focalisée sur l'analyse économique des impacts que les réformes et les changements sociétaux ont eus tant sur le fonctionnement des exploitations que sur le revenu des agriculteurs. Cela s'explique pour partie par le fait qu'en France, l'agriculture est un secteur économique à la fois stratégique et protégé par la PAC. Ce contexte a placé les agriculteurs dans une situation de concurrence masquée, avec des systèmes de prix garantis qui ont sans doute limité l'intérêt des agriculteurs pour des démarches stratégiques visant à rechercher continuellement un avantage concurrentiel, au contraire de pays plus libéraux davantage enclins à considérer l'agriculture à l'instar de n'importe quel autre secteur de leur économie. Toutefois, des dispositifs de formation à la stratégie d'entreprise à destination des agriculteurs commencent à être diffusés (sans qu'ils soient pour autant issus de travaux de recherche en sciences de gestion) (PERVANCHON et al., 2007 ; GOULET et al., 2008).

\section{CONCLUSION}

Face à leurs évolutions internes et aux évolutions externes contemporaines, les exploitations agricoles doivent sans cesse s'adapter et développer une stratégie leur permettant d'assurer leur pérennité. Nous pensions que nous allions observer une montée en puissance des travaux en gestion sur le pilotage stratégique, sur le management du risque, sur celui de la qualité ou encore sur l'amélioration continue des performances visant à conserver un avantage concurrentiel. Mais il n'en a rien été.

En effet, l'analyse bibliométrique des documents référencés dans les bases de données BDESR, BDSAD et ProdINRA publiés entre 1990 et 2008 montre que les travaux concernant la gestion de l'exploitation agricole sont en déclin et que ceux qui ont été produits sur la dernière période (2000-2008) n'abordent que très rarement la question des pratiques de management stratégique. Mentionnons à ce propos que, sur cette période, le management stratégique n'est abordé qu'au travers de quelques rares travaux consacrés au management environnemental. On ne trouve que peu de travaux faisant état du farm management ou s'y consacrant, tels que ceux développés par les AngloSaxons. Vraisemblablement, l'analyse économique de la production agricole, puis celle de la décision de l'agriculteur et, enfin, celle de la socio-économie agricole ont monopolisé l'attention des chercheurs. En un certain sens, on peut défendre l'idée que le renouvellement des courants théoriques en gestion de l'exploitation agricole n'a pas été complet puisqu'il a occulté en partie les travaux consacrés au management stratégique de l'exploitation agricole.

Nous défendons l'idée que la démarche stratégique devrait être inscrite à l'ordre du jour scientifique pour être discutée, éprouvée et amendée, car elle devrait finir par s'imposer, comme le laissent pressentir la dérégulation des marchés, l'exacerbation de la concurrence entre exploitations, le développement de la certification environnementale des exploitations prévue par le Grenelle de l'Environnement ou encore la montée en puissance du développement durable et de la responsabilité sociétale des entreprises (RSE). En cela, il nous semble que la démarche de management stratégique pourrait s'affirmer, à l'avenir, comme un levier d'amélioration continue de la performance globale de l'exploitation agricole. La démarche de réflexion stratégique menée par l'agriculteur permet d'avoir une vision partagée (entre parties prenantes) de la situation de l'exploitation et de son projet. Le choix et la mise en ouvre d'outils de pilotage stratégique, comme par exemple le tableau de bord stratégique (KAPLAN et NORTON, 1992), permettent d'aborder la question de la durabilité de l'exploitation en mobilisant une vision systémique s'appuyant sur des indicateurs de performance qui peuvent ne plus être uniquement financiers. En effet, cette approche intègre certains éléments qualitatifs, tels que des indicateurs liés au processus de production et d'apprentissage ou des caractéristiques organisationnelles de l'exploitation. Ces éléments permettent d'intégrer la dimension immatérielle de l'entreprise pour expliquer l'origine de sa performance en vue, au final, de permettre à l'agriculteur de mieux s'adapter au changement et de pérenniser son exploitation.

\section{BIBLIOGRAPHIE}

AMELINCKX (J.) \& BARRAS (L.), Agriculteur : le sens du métier, CER France - Les Cahiers du CER, 48 p., 2002. BARNARD (C.S.) \& NIX (J.), Farm planning and control, Cambridge, New York, Cambridge University Press, 600 p., 1979. 
BARTHÉLÉMY (D.), Évaluer l'entreprise agricole, Paris, PUF, 229 p., 1997.

BAUDRY (J.) \& ThENAIL (C.), « La nécessaire articulation de l'écologie et de l'agronomie pour la gestion des paysages agricoles", Comptes rendus de l'Académie d'agriculture de France, 87(4), pp. 155-163, 2001.

BENOîT (M.), "La Conversion en agriculture biologique : importance du contexte et incidences économiques ", Bulletin des GTV (Groupements Techniques Vétérinaires), Hors-série « Élevage et agriculture biologique », pp. 27-31, 2001.

Brossier (J.), Chia (E.), Marshall (E.) \& Petit (M.), Gestion de l'exploitation agricole familiale: éléments théoriques et méthodologiques, Dijon, ENESAD/CNERTA, 215 p., 1997.

CAPT (D.), Demande de biens différenciés, comportements spatiaux et diversification de l'activité des exploitations agricoles, Document de recherche $\mathrm{n}^{\circ} 45$ (Série Thèses), Université de Bourgogne, Enesad et Inra, Dijon, 366 p., 1994.

CASAVANT (K.L.) \& Infanger (C.L.), Agricultural Economics \& Management, Upper Saddle River, New Jersey, Prentice Hall, 434 p., 1999.

Chatelin (M.H.) \& Poussin (J.C.) (1991), "Diagnostic d'exploitation agricole: apport de la simulation ", Économie rurale 206, pp. 77-80.

Chombart de Lauwe (J.) \& PoiteVin (J.), Gestion des exploitations agricoles, Paris, Dunod, 222 p., 1957. Chombart de Lauwe (J.), Poitevin (J.) \& Tirel (J.-C.), Nouvelle gestion des exploitations agricoles, Paris, Dunod, 507 p., 1963.

Coleno (F.C.) \& Duru (M.), « L'apport de la gestion de production aux sciences agronomiques ", Natures Sciences Sociétés 13(3), pp. 247-257, 2005.

COLIN (J.-P.), «Farm management versus production economics: de l'actualité d'un vieux débat américain ", Les Cahiers de la Recherche Développement 27, pp. 85-93, septembre 1990.

Colson (F.) \& Pineau (B.), "Les Indicateurs de détection de la difficulté financière des exploitations en période d'installation ", Économie rurale 206, pp. 57-63, 1991.

Debaeke (P.), Willaume (M.), Casadebaig (P.) \& Nolot (J.M.), «Raisonner les systèmes de culture en fonction de la disponibilité en eau ", Innovations agronomiques 2, pp. 19-36, 2008.

Drouet (D.) \& Boullet (P.) (2006), Le Concept d'exploitation agricole a-t-il encore un avenir?, CER France, Les Cahiers du CER, 28 p., 2006.

DURU (M.), «Les prairies multi-spécifiques : vers la troisième révolution fourragère ? Bilan des journées et pistes de travail », Fourrages 195, pp. 331-342, 2008. Girard (N.), Havet (A.), Chatelin (M.H.), Gibon (A.), Hubert (B.) \& Rellier (J.P.), "Formalisation des relations entre stratégie et pilotage dans les systèmes fourragers. Propositions pour la conception d'instruments d'aide à la décision : recherches-systèmes en agriculture ", Les Cahiers de la recherche-développement 39, pp. 60-72 et 97-100, 1994.

Goulet (F.), Pervanchon (F.), Conteau (C.) \& Cerf (M.), "Les Agriculteurs innovent par euxmêmes pour leurs systèmes de culture ", in REAU (R.) \& DORE (T.), Systèmes de culture innovants et durables, Dijon, Educagri Éditions, pp. 53-69, 2008.

Grolleau (G.), « Management environnemental et exploitation agricole ", Économie rurale 262, marsavril, pp. 35-47, 2001.

GuiCHARD (M.) \& Michaud (R.), La Stratégie à pas comptés : piloter l'entreprise agricole dans l'incertitude et dans la complexité, CNERTA-SED, 298 p., 1994.

Hemidy (L.) \& Soler (L.-G.), « Nouvelles exigences en gestion de la petite entreprise agricole : réflexions à partir du cas français ", Revue Internationale PME, pp. 83-102, 1992.

Hemidy (L.) \& SOlER (L.-G.), Un modèle conceptuel pour le pilotage stratégique de l'entreprise agricole, Communication pour le $38^{\mathrm{e}}$ séminaire EAAE, 35 octobre 1994, Copenhague, 14 p., 1994.

Hubert (B.), "Combiner les analyses économiques, techniques et écologiques pour étudier les conditions du développement local ", in Systèmes d'élevage et gestion de l'espace en montagnes et collines méditerranéennes, GibON (A.), LASSEUR (J.), MANRIQUe (E.) \& Masson (P.), 1999.

Institut National de la Recherche Agronomique, Schéma stratégique du département Économie et Sociologie rurales, juillet 1999-juillet 2003, version préparée pour les directoriales de juin 1999, 40 p. http:/www.inra.fr/internet/Departements/ESR/depa rtement/ssd-esr-9903.pdf?-PHPSESSID=76f85105198db7156a9f0eac8fcb8bd

Pluvinage (J.) \& Revilla (R.) (eds), pp. 9-14.

Kaplan (R. S.) \& NorTON (D. P.), "Balanced scorecard - Measure that drive performance", Harvard Business Review, pp. 71-79, january-february 1992.

KAY (R.D.), Farm management: planning, control, and implementation, $2^{\text {nd }}$ ed., New York, McGraw-Hill, 401 p., 1986.

LAURENT (C.) \& REMY (J.), "L'Exploitation agricole en perspective ", Courrier de l'environnement de I'INRA 41, pp. 5-24, 2000.

Marchesnay (M.), "Le Conseil d'entreprise appliqué aux petites entreprises : quels enseignements pour l'entreprise agricole?", Revue des chambres d'agriculture 826, pp. 5-10, 1994.

Marchesnay (M.), "Petite entreprise et entrepreneur", in Encyclopédie de gestion, SIMON (Y.) \& Joffre (P.) (dir.), Paris, Économica, pp. 2209-2219, 1997.

Marshall (E.), BonneViale (J.-R.) \& FrancForT (I.), Fonctionnement et diagnostic global de l'exploitation agricole. Une méthode interdisciplinaire pour la formation et le développement, Dijon, ENESAD-SED, 174 p., 1994. 
Maze (A.), Aubry (C.) \& PAPY (F.), « La certification des exploitations agricoles ", Économie rurale 258, pp. 134-139, 2000.

MintzBerg (H.), The Rise and Fall of Strategic Planning: Reconceiving the Roles for Planning, Plans, Planners, New York, The Free Press, 1994.

Noell (C.) \& Lund (M.), The balances Scorecard (BSC) for Danish Farm - Vague framework or functional instrument, Farm management - Proceedings of NJF Seminar, n³45, Oslo, 2-4 october 2002, pp. 187-204, 2002.

Olson (K.D.), Farm Management: Principles and Strategies, Iowa, Blackwell Publishing Co, 429 p., 2004. PERVANCHON (F.), "Accompagner les agriculteurs dans leur stratégie d'entreprise ", Fourrages (192), pp. 511-516, 2008.

REMY (J.), "Révéler ou construire la demande sociale? Les contrats territoriaux d'exploitation", Compte rendus de l'Académie d'Agriculture de France 88 (7), pp. 67-77, 2002.

Seronie (J.-M.) \& Boullet (P.) (2007), L'Exploitation agricole flexible, CER France - Les Cahiers du CER, 36 p., 2007.

Thannberger (E.), "Contrat agri-environnemental : un outil pour quelles coordinations?", Économie rurale 249, Les mesures agri-environnementales, pp. 34-40, 1999.

TURner (J.) \& TAYLOR (M.), Applied farm management, Oxford, Malden, Mass, Blackwell Science, 394 p., 1998. 